\title{
Headspace passive dosing of volatile hydrophobic organic chemicals from a lipid donor - linking their toxicity ot well-defined exposure for an improved risk assessment
}

Trac, Ngoc Lam; Nørgaard Schmidt, Stine; Holmstrup, Martin; Mayer, Philipp

Published in:

Environmental Science and Technology

Link to article, DOI:

10.1021/acs.est.9b04681

Publication date:

2019

Document Version

Peer reviewed version

Link back to DTU Orbit

Citation (APA):

Trac, N. L., Nørgaard Schmidt, S., Holmstrup, M., \& Mayer, P. (2019). Headspace passive dosing of volatile hydrophobic organic chemicals from a lipid donor - linking their toxicity ot well-defined exposure for an improved risk assessment. Environmental Science and Technology, 53(22), 13468-13476.

https://doi.org/10.1021/acs.est.9b04681

\section{General rights}

Copyright and moral rights for the publications made accessible in the public portal are retained by the authors and/or other copyright owners and it is a condition of accessing publications that users recognise and abide by the legal requirements associated with these rights.

- Users may download and print one copy of any publication from the public portal for the purpose of private study or research.

- You may not further distribute the material or use it for any profit-making activity or commercial gain

- You may freely distribute the URL identifying the publication in the public portal 


\title{
HEADSPACE PASSIVE DOSING OF VOLATILE HYDROPHOBIC ORGANIC CHEMICALS FROM A LIPID DONOR - LINKING THEIR TOXICITY TO WELL-DEFINED EXPOSURE FOR AN IMPROVED RISK ASSESSMENT
}

\author{
Lam Trac, Stine N. Schmidt, Martin Holmstrup, and Philipp Mayer \\ Environ. Sci. Technol., Just Accepted Manuscript • DOI: 10.1021/acs.est.9b04681 • Publication Date (Web): 15 Oct 2019 \\ Downloaded from pubs.acs.org on October 21, 2019
}

\section{Just Accepted}

"Just Accepted" manuscripts have been peer-reviewed and accepted for publication. They are posted online prior to technical editing, formatting for publication and author proofing. The American Chemical Society provides "Just Accepted" as a service to the research community to expedite the dissemination of scientific material as soon as possible after acceptance. "Just Accepted" manuscripts appear in full in PDF format accompanied by an HTML abstract. "Just Accepted" manuscripts have been fully peer reviewed, but should not be considered the official version of record. They are citable by the Digital Object Identifier (DOI®). "Just Accepted" is an optional service offered to authors. Therefore, the "Just Accepted" Web site may not include all articles that will be published in the journal. After a manuscript is technically edited and formatted, it will be removed from the "Just Accepted" Web site and published as an ASAP article. Note that technical editing may introduce minor changes to the manuscript text and/or graphics which could affect content, and all legal disclaimers and ethical guidelines that apply to the journal pertain. ACS cannot be held responsible for errors or consequences arising from the use of information contained in these "Just Accepted" manuscripts. 
1 HEADSPACE PASSIVE DOSING OF VOLATILE HYDROPHOBIC ORGANIC CHEMICALS FROM A LIPID DONOR 2 LINKING THEIR TOXICITY TO WELL-DEFINED EXPOSURE FOR AN IMPROVED RISK ASSESSMENT

3 Lam Ngoc Trac ${ }^{1}$, Stine Nørgaard Schmidt ${ }^{1}$, Martin Holmstrup ${ }^{2}$, Philipp Mayer *1

$4{ }^{1}$ Department of Environmental Engineering, Technical University of Denmark, DK-2800 Kgs. Lyngby,

5 Denmark

$6 \quad{ }^{2}$ Department of Bioscience, Aarhus University, DK-8600 Silkeborg, Denmark

\section{AUTHOR INFORMATION}

8 Corresponding author

$9 \quad$ *Phone: +45 4525 1569; fax: +45 4593 2850; e-mail: philm@env.dtu.dk

10 Current affiliation for Stine Nørgaard Schmidt: National Research Council, Atlantic Ecology Division NHEERL

11 /ORD, U.S. Environmental Protection Agency, Narragansett, Rhode Island 02882, USA.

\section{Notes}

13 The authors declare no competing financial interest. 


\section{ABSTRACT} Raoult's law.

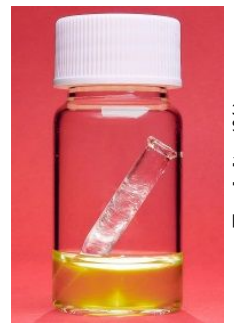

Algal growth inhibition

High hydrophobicity and volatility of chemicals often lead to substantial experimental challenges, but was here utilized in headspace passive dosing (HS-PD) to establish and maintain exposure: The pure chemical served as passive dosing donor for controlling exposure at saturation, whereas triglyceride oil containing the chemical was used to control lower exposure levels. These donor solutions were added to glass inserts placed in the closed test systems. Mass balance calculations confirmed a dominant donor capacity for all chemicals except isooctane. This HS-PD method was applied to algal growth inhibition and springtail lethality tests with terpenes, alkanes, and cyclic siloxanes. Headspace concentrations above the lipid donors were measured for three chemicals to determine their chemical activity, using saturated vapor as analytical standard and thermodynamic reference. Toxicity was related to chemical activity and calculated concentrations in membranes at equilibrium with the lipid donor. For both tests and all chemicals, toxic effects were observed within or above the reported range for baseline toxicity, meaning that no excess toxicity was observed. The toxicity of siloxanes was markedly higher to the terrestrial springtail than the aquatic algae, which is consistent with a more efficient mass transfer of these volatile hydrophobic chemicals in air compared to water.

Keywords: Raphidocelis subcapitata, Folsomia candida, aliphatic hydrocarbons, narcosis, Henry's law,

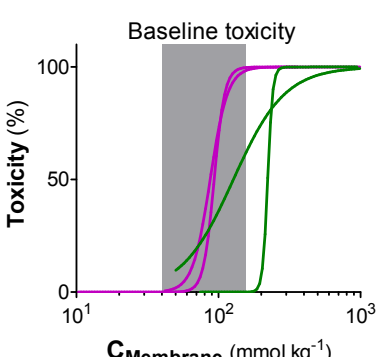

C $_{\text {Membrane }}\left(\mathrm{mmol} \mathrm{kg}^{-1}\right)$

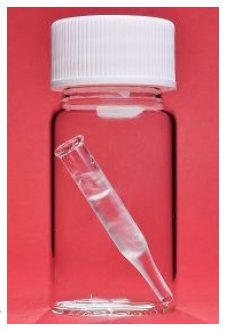

Springtail lethality 


\section{INTRODUCTION}

High volatility and high hydrophobicity make chemicals "difficult to test" with regards to toxicity testing ${ }^{1}$. When a chemical possesses both properties, it becomes almost untestable with current standard toxicity test methods. In fact, for volatile hydrophobic chemicals with an air-water partition coefficient $\left(K_{\mathrm{Aw}}\right)$ greater than $1(\mathrm{~L} / \mathrm{L})$, a considerable mass fraction will partition into the headspace of aquatic test systems $^{2,3}$. The exposure via soil interstitial air will become important for terrestrial organisms, because of a more efficient mass transfer via air compared to water ${ }^{4-6}$. For both aquatic and terrestrial testing, it is generally challenging to first establish well-defined exposure at the beginning of the test, then to maintain constant exposure during the test, and finally to analytically confirm this exposure ${ }^{1}$. Experimental strategies to cope with these challenges include (1) flow-through systems with a continuous supply of spiked medium $^{1},(2)$ non-depletive testing focusing at minimizing test substance losses ${ }^{2}$, and (3) passive dosing where the concentration in the test is established and re-supplied by phase partitioning from a pre-loaded polymer ${ }^{7}$. Recently, a new headspace passive dosing (HS-PD) approach was developed for enabling aquatic toxicity testing of volatile hydrophobic organic chemicals (VHOCs) at the maximum exposure level ${ }^{8}$. The toxicity tests using HS-PD were conducted in gas tight vials, and the pure liquid test substance was used as a partitioning donor to establish and control the test exposure exactly at the saturation level ${ }^{8}$. The present study was directed at developing and applying a HS-PD approach for the dose-response testing of VHOCs.

The exposure to a test substance can be expressed in several ways that go beyond the concentration in the test medium (e.g., air and water). Various approaches have been proposed to link adverse biological responses to the internal exposure, such as the critical body residue model ${ }^{9}$, critical body burdens ${ }^{10}$, toxic cell concentrations ${ }^{11}$, and the target lipid model ${ }^{12}$. These studies advocate the use of the critical internal dose that causes $50 \%$ mortality in test organisms, e.g., critical body residue CBR-50, for the interpretation and prediction of toxicity. This is due to the fact that external medium-based effective concentrations vary greatly for narcotic chemicals, whereas their CBRs remain within a much more narrow range $\mathrm{e}^{9,13}$. It has been 
reported that nonpolar narcosis initiates at a target concentration range of $40-160 \mathrm{mmol} / \mathrm{kg}$ membrane lipid $^{10}$, which in the present study is used as a reference for identifying excess toxicity.

Another way to interpret and study the toxicity of narcotic chemicals is to link toxic effects to chemical activity $^{14,15}$. Chemical activity expresses the chemical potential relative to a chosen reference state ${ }^{16-19}$, which for hydrophobic chemicals often is the pure chemical in its liquid state where chemical activity is set to unity ${ }^{16}$. The chemical activity is then defined between 0 and 1 , and differences in chemical activities between different phases drive the diffusion, sorption, and partitioning of the chemical, with a direction from high to low chemical activity ${ }^{16}$. Therefore, chemical activity can give new insights and facilitate comparison of chemical exposures across environmental compartments including air, water, soil, sediment, and biota. The relationship between chemical activity and toxicity was first proposed by Ferguson in the late 1930 ' $\mathrm{s}^{14}$, and several studies have since shown that nonpolar narcotic chemicals exert baseline toxicity when reaching a chemical activity between $0.01-0.1^{15,20-22}$. Specific toxicity or excess toxicity is thus indicated when effects occur at a lower chemical activity $(<0.001)^{19,23}$.

The link between the toxicity of hydrophobic chemicals and internal exposure or chemical activity has been explored more recently with the use of passive dosing, which allows precise exposure control7,13,20,24-30. Since passive dosing is based on equilibrium partitioning of the test chemical between different phases, its application in toxicity tests has several advantages: (1) by controlling concentrations in the donor, the concentrations in the test medium are precisely controlled, (2) the theoretical equilibrium partitioning concentration of test chemical in the organisms can be estimated, and (3) the chemical activity in the test system is determined by the concentration in the donor and can be measured in the headspace.

The present study introduces the use of a lipid as passive dosing donor for the toxicity testing of liquid VHOCs at and below their saturation level. Lipid has recently been successfully applied as a partitioning donor in dedicated analytical and partitioning studies with semivolatile organic chemicals ${ }^{31-33}$, while other scientists used vacuum pump oil as partitioning donor for controlling headspace concentrations in 
autosampler vials for calibration purposes ${ }^{34,35}$. The working principle of the new passive dosing method is based on equilibrium partitioning between donor and exposure medium, without a direct contact between these two phases. The test system is simple: The lipid donor is loaded by direct addition of the liquid test chemical and then used for establishing and controlling exposures below the saturation level, while the test chemical in the pure liquid form is used to establish and control exposure at the saturation level. The donor in this passive dosing approach is kept separate from the test medium and organisms using a glass insert (see the TOC Figure). We used purified plant oil as lipid donor, which is a medium-chain triglyceride of fractionated plant fatty acids, e.g., caprylic (C8) and capric (C10) fatty acids. There are a number of advantages when using such a liquid lipid as donor: (1) it is easy to load these high capacity donors to different chemical concentrations for toxicity testing by directly mixing the test chemical with the purified plant oil, (2) it allows measurements of chemical activity based on headspace concentrations of test chemicals, (3) it allows prediction of "concentrations in membranes at equilibrium with the donor" based on estimated storage lipid to membrane lipid partition coefficients, and (4) toxicity can finally be linked to both chemical activity and calculated equilibrium concentrations in membranes.

The aims of the present study were: (1) to develop a passive dosing method that can control the exposure to VHOCs at and below their saturation level, (2) to apply the method to dose-response testing of mono terpenes, alkanes, and cyclic volatile methyl siloxanes with the freshwater green algae Raphidocelis subcapitata (Sphaeropleales; Selenastraceae) and the terrestrial springtail Folsomia candida (Collembola; Isotomidae), and (3) to link the toxic effects to calculated equilibrium concentrations in membranes and to the measured chemical activities, and thus assess whether the test chemicals exert baseline or excess toxicity. The hypotheses were that: (i) the HS-PD approach can control exposure concentrations at and below the saturation level in algal growth inhibition test and springtail lethality test, (ii) the tested chemicals do not exert excess toxicity, and (iii) since chemicals with high $K_{\text {ow }}$ and high $K_{\text {AW }}$ have more efficient mass transfer in air compared to water, such chemicals can have faster uptake kinetics in the absence of water and thus higher toxicity in small terrestrial compared to aquatic organisms. 


\section{MATERIALS AND METHODS}

2.1. Chemicals and materials

The following test chemicals were from Sigma-Aldrich Denmark with purity $\geq 99 \%$ unless otherwise stated: $\alpha-(+)-$ pinene, $\alpha-(-)-$ pinene, S-(-)-limonene (96\%), octamethylcyclotetrasiloxane (D4) (98\%), decamethylcyclopentasiloxane (D5) (97\%), isooctane, n-nonane, n-undecane (Sigma-Aldrich, Germany), n-dodecane and isododecane (98\%) (TCI Chemicals, Belgium), and n-tridecane (Fluka AG, Switzerland). Miglyol 812 purified plant oil was obtained from Cremer Oleo GmbH, Germany.

\subsection{Headspace passive dosing using lipid as donor}

\subsubsection{Headspace passive dosing system}

Test chemicals were weighed and mixed into Miglyol oil (without any pre-treatment of the oil) to obtain desired test concentrations (\% $\mathrm{w} / \mathrm{w}$, mass $\%$ ), while pure liquid test chemicals with no further treatment were used to control exposure at the saturation level according to Trac and colleagues ${ }^{8}$. An amount of 0.2 $\mathrm{mL}$ of the donor solutions was added to $0.3-\mathrm{mL}$ glass inserts embedded with glass wool to increase the surface area for the passive dosing. The inserts were then placed in $20-\mathrm{mL}$ test vials containing $4 \mathrm{~mL}$ algal test medium or in 20-mL empty test vials for the springtail lethality experiment (further details in Section 2.3 and depicted in the TOC Figure). The test vials were then closed air tight with screw caps with Teflonlined (PTFE) septa, and finally the complete systems were kept at $20 \pm 1{ }^{\circ} \mathrm{C}$ for $24 \mathrm{~h}$ for pre-equilibration. During this period, the closed test vials were shaken at $200 \mathrm{rpm}$ (10 $\mathrm{mm}$ orbit) in order for the test chemicals to equilibrate quickly within the test system. Shaking of test vials was not necessary for springtail lethality tests. At test start, the pre-equilibrated vials were opened shortly for adding test organisms. The loss of test chemical during the opening was assumed to be limited to the fraction in the headspace, which was negligible as demonstrated by the mass balance calculations below.

\subsubsection{Mass balance calculations}


129 Mass balance calculations were done for an algal toxicity test system with and without passive dosing

130 donor (Table 1) in order (1) to illustrate the differences between the two systems and (2) to demonstrate

131 that the donor capacity was sufficient for controlling the partitioning within the closed system (i.e.,

132 negligible donor depletion). The calculations were done for all test chemicals using partition coefficients

133 from the UFZ-LSER database ${ }^{36}$, a donor volume of $200 \mu \mathrm{L}$, a water volume of $4 \mathrm{~mL}$, and a vial volume of 20

$134 \mathrm{~mL}$ (see Section 2.2.1).

135 Table 1: Partition coefficients of the test chemicals and their mass balances calculated for test systems with

136 and without a passive dosing donor. PD: passive dosing, OW: octanol-water, AW: air-water, LW: storage

137 lipid-water, MW: membrane lipid-water, LA: storage lipid-air.

\begin{tabular}{|c|c|c|c|c|c|c|c|c|c|c|}
\hline \multirow[b]{2}{*}{ Compound } & \multicolumn{5}{|c|}{ Governing partition coefficient } & \multicolumn{2}{|c|}{$\begin{array}{l}\text { Mass balance } \\
\text { without PD }\end{array}$} & \multicolumn{3}{|c|}{$\begin{array}{c}\text { Mass balance } \\
\text { with PD }\end{array}$} \\
\hline & $\begin{array}{r}\text { Log } \\
K_{\mathrm{OW}}{ }^{a} \\
\end{array}$ & $\begin{array}{r}\log \\
K_{\mathrm{AW}}{ }^{a} \\
\end{array}$ & $\begin{array}{l}\mathrm{Log} \\
K_{\mathrm{LW}}{ }^{a} \\
\end{array}$ & $\begin{array}{r}\text { Log } \\
K_{\mathrm{MW}}{ }^{a} \\
\end{array}$ & $\begin{array}{l}\mathrm{Log} \\
K_{\mathrm{LA}}^{b} \\
\end{array}$ & Air & Water & Lipid & Air & Water \\
\hline n-nonane & 5.46 & 2.38 & 5.72 & 4.96 & 3.34 & $99.9 \%$ & $0.10 \%$ & $96.5 \%$ & $3.49 \%$ & $<0.01 \%$ \\
\hline n-undecane & 6.57 & 2.69 & 6.86 & 5.94 & 4.17 & $99.9 \%$ & $0.05 \%$ & $99.5 \%$ & $0.53 \%$ & $<0.01 \%$ \\
\hline n-dodecane & 7.13 & 2.84 & 7.43 & 6.43 & 4.59 & $100.0 \%$ & $0.04 \%$ & $99.8 \%$ & $0.20 \%$ & $<0.01 \%$ \\
\hline n-tridecane & 7.68 & 3.01 & 7.98 & 6.90 & 4.97 & $100.0 \%$ & $0.02 \%$ & $99.9 \%$ & $0.08 \%$ & $<0.01 \%$ \\
\hline isooctane & 4.65 & 2.40 & 4.81 & 4.19 & 2.41 & $99.9 \%$ & $0.10 \%$ & $76.5 \%$ & $23.50 \%$ & $0.02 \%$ \\
\hline isododecane & 6.65 & 3.19 & 6.79 & 5.89 & 3.60 & $100.0 \%$ & $0.02 \%$ & $98.1 \%$ & $1.95 \%$ & $<0.01 \%$ \\
\hline Limonene & 4.44 & 0.54 & 4.60 & 4.09 & 4.06 & $93.3 \%$ & $6.73 \%$ & $99.3 \%$ & $0.68 \%$ & $0.05 \%$ \\
\hline$\alpha$-pinene & 4.61 & 1.23 & 4.83 & 4.24 & 3.60 & $98.5 \%$ & $1.45 \%$ & $98.0 \%$ & $1.95 \%$ & $0.03 \%$ \\
\hline D4 & 6.92 & 3.21 & 6.61 & 5.65 & 3.40 & $100.0 \%$ & $0.02 \%$ & $97.0 \%$ & $3.05 \%$ & $<0.01 \%$ \\
\hline D5 & 8.07 & 3.52 & 7.51 & 6.39 & 3.99 & $100.0 \%$ & $0.01 \%$ & $99.2 \%$ & $0.80 \%$ & $<0.01 \%$ \\
\hline
\end{tabular}

${ }^{a}$ data from UFZ-LSER database ${ }^{36}$

$139{ }^{b}$ calculated by subtracting Log $K_{\mathrm{LW}}$ by Log $K_{\mathrm{AW}}$

$140{ }^{c}$ due to rounding numbers may add up to more or less than $100 \%$

141 The mass balance calculations in Table 1 apply to the Henry's law regime with donor concentrations up to

$14230 \%$, which complements the mass balance calculations for the saturation level in Trac et al. ${ }^{8}$. In the air-

143 water system, all chemicals predominantly partition into the headspace, which asks for and justifies 
exposure confirmation to be done by headspace analysis. In the passive dosing system, $200 \mu \mathrm{L}$ of storage lipid was found to have sufficient donor capacity for the passive dosing of water and headspace with donor depletion $<10 \%$ for all chemicals except isooctane. The high capacity of the Miglyol oil is consistent with lipid-water partition coefficients being considerably higher than $K_{\mathrm{OW}}$ and $K_{\mathrm{Mw}}$. The donor depletion in the Henry regime (Table 1 ) is higher than in the saturation regime, and can thus be used as a conservative estimate.

\subsubsection{Analytical exposure confirmation}

This experiment was performed to (1) analytically determine the chemical activity of three test chemicals in the HS-PD system relative to their saturation level, and (2) link the test chemical concentrations in the lipid donor to chemical activity and thus determine the actual chemical activity of these test chemicals in the toxicity tests. This exposure analysis was performed for limonene, n-nonane, and D4, chosen as representatives for the chemical groups tested. A series of concentrations in Miglyol oil (mass \%) was prepared for each chemical, namely, limonene: 100, 89.9, 79.9, 40, 20, 10.6, 5.1, 2.5, 1.7\%; n-nonane: 100, $89.9,80,40,20,10,5,2.5,1 \%$; and D4: 100, 95.5, 89.9, 79.9, 40, 19.9, 10, 4.9, 2.5\%. Two $\mathrm{mL}$ of each concentration solution was transferred into a $20-\mathrm{mL}$ autosampler vial, which was then closed air tight and left in darkness at $20 \pm 1^{\circ} \mathrm{C}$ for $24 \mathrm{~h}$ for equilibration. Fully automated headspace sampling was performed on the test vials utilizing an autosampler, and the samples were injected on a GC-MS to measure the chemical concentrations in the vapor phase of the HS-PD system relative to the saturated vapors above the pure test substances. The experiment was performed in triplicate.

We used the pure liquid chemical as analytical standard and as the thermodynamic reference $\left(a_{\text {saturation }}=1\right)$. Therefore, the chemical activity $(a)$ in the test system was determined by dividing the headspace measurement of that system (peak area) by the headspace measurement above the pure test chemical, after confirming the linearity of the MS by separate measurements.

\subsubsection{Headspace GC-MS}


168 Headspace samples were initially taken manually and injected on a GC-FID, which however provided 169 insufficient analytical precision. Headspace samples were then taken with a CTC PAL RSI 85 autosampler 170 (CTC Analytics, Zwingen, Switzerland) and injected directly onto an Agilent Technologies GC-MSD system 171 (7890B/5877A GC/MSD). On the autosampler, the samples were equilibrated at $35^{\circ} \mathrm{C}$ at intermittent orbital shaking for 10 minutes before $100-\mu \mathrm{L}$ samples were withdrawn from the headspace of the test vials. All analyses were run with a split ratio of 100:1, a DB-5ms Ultra inert column (60 $\mathrm{m} \times 250 \mu \mathrm{m} \times 0.25 \mu \mathrm{m})$, the following temperature program: initial: $80^{\circ} \mathrm{C}$, rate: $20^{\circ} \mathrm{C} / \mathrm{min}$, final: $210^{\circ} \mathrm{C}$, and in $\mathrm{TIC}$ scanning mode with a mass-to-charge range from 40-200 m/z. TIC peak areas of test chemicals were manually integrated (MSD Chemstation, Agilent Technologies Inc.) and used for the determination of chemical activity. To ensure that the measured peak areas throughout the entire concentration range were within the linear range of the MS detector, an additional analysis was performed in duplicate for each test chemical with a split ratio of 10:1 (Figure S1). While this exposure analysis based on headspace GC-MS analysis proved to be simple, effective, and precise, it required still about 40 measurements for each of the three chosen chemicals including the important linearity check of the MS detector.

\subsection{Toxicity tests}

\subsubsection{Algal growth inhibition test}

The HS-PD approach was applied to control exposure at and below the saturation level. The test chemicals were $\alpha-(+)$-pinene, $\alpha-(-)$-pinene, limonene, $n$-nonane, $n$-undecane, and $n$-tridecane. Six concentrations were prepared for each test chemical (Table S4). The donor solutions and test vials were prepared, and a pre-equilibration was completed as described in Section 2.2.1. The $R$. subcapitata growth inhibition tests were conducted as described earlier ${ }^{8}$. Each treatment, including the oil control (insert with Miglyol oil), was performed in 3 replicates, and the global control (without insert and oil) was performed in 6 replicates. A lipid content of $17-19.5 \%$ (w/w, dry weight) has been reported for $R$. subcapitata. ${ }^{37,38}$

\subsubsection{Acute springtail lethality test}


192 The springtail F. candida was cultured in closed Petri dishes containing a cast of a mixture of charcoal and 193 plaster of Paris (1:8). The cast was moistened, and dried yeast was used for feeding. The cultures were 194 maintained at $20 \pm 1{ }^{\circ} \mathrm{C}$ with a $12: 12 \mathrm{~h}$ light:dark regime. The springtails had an average fresh weight of approximately $110 \mu \mathrm{g}$ and were picked randomly from different Petri dishes for toxicity tests. The lipid content of $F$. candida has been reported to be $9.9 \%$ dry weight. ${ }^{20}$ A range of different mass concentrations (\%) of each test chemical was prepared in Miglyol oil (Table S4), and the HS-PD was applied to control the exposure in the test vials as described in Section 2.2.1. The test chemicals were $\alpha-(+)$-pinene, limonene, D4, D5, isooctane, n-nonane, n-dodecane, and isododecane. At test start, $2 \mu \mathrm{L}$ Milli-Q water was added to the bottom center of the pre-equilibrated test vial to ensure sufficient humidity during the test period. Ten animals were transferred to each vial and most of them were observed on the bottom of the test vial throughout the test. The uptake of test chemicals via the water was negligible due to the small water volume, low aqueous concentrations, and limited water uptake by the animals. Hence, the exposure through air was the only uptake route of test chemicals in this HS-PD system. The springtail lethality test was conducted at $20 \pm 1{ }^{\circ} \mathrm{C}$ with a $12: 12 \mathrm{~h}$ light:dark photoperiod. Ten global control vials (with insert only, in this case) and 10 oil control vials (insert with Miglyol oil) were included in the experiment. Three replicates were done for each treatment. After 7 days of exposure, springtails were transferred to clean Petri dishes with a clean and moistened cast of charcoal and plaster of Paris mixture and allowed to recover for $24 \mathrm{~h}$. The lethality was then examined, and springtails were characterized as dead when not able to move any part of their body, after gentle stimulation with a fine brush. In all tests, the lethality of the global controls and oil controls was less than $10 \%$, which confirmed the validity of the springtail tests.

2.4. Data treatment

2.4.1. Exposure confirmation experiment

The relationship between test chemical concentration in the lipid donor and chemical activity was established by plotting the measured chemical activity $a$ as a function of mass concentration of chemical in 
216 Miglyol oil (X, \%) and fitting the data with a one-phase association exponential model using least-squares 217 regression by GraphPad Prism 5.0 software (GraphPad Software, Inc., USA):

$$
a=a_{\max } \times\left(1-e^{-k \times X}\right)
$$

\subsubsection{Toxicity experiments}

In the algal growth inhibition test with terpenes, there was no significant difference in algal biomass between the global controls and the oil controls after $72 \mathrm{~h}$ (Figure S2A). In the test with alkanes, there was a minor but statistically significant difference $(p<0.05$, Figure $S 2 B)$. And because the majority of the test solutions were prepared in Miglyol oil, we used the mean growth rate of the oil control samples as the reference for calculating the inhibition caused in the treatments. The growth inhibition was calculated as described earlier ${ }^{8}$. Growth inhibition that was numerically higher than $100 \%$ was set to a value of $100 \%$ (i.e., full inhibition) for all further data analyses. The concentration of the test chemicals in the donor $\left(C_{\text {Donor }}\right)$ was calculated as the added mass of chemical relative to the total mass of chemical and Miglyol oil, and concentrations were expressed in units of \% (mass:mass) or mmol/ $\mathrm{kg}$ :

$$
C_{\text {Donor }}=\left(W_{C} / M W\right) /\left(W_{C}+W_{\text {oil }}\right)
$$

Where $W_{C}$ is the added mass $(\mathrm{g})$ and $M W$ the molecular weight $\left(\mathrm{g} \mathrm{mol}^{-1}\right)$ of the test chemical, and $W_{o i l}$ is the added mass (g) of Miglyol oil.

The actual chemical activities of limonene, n-nonane, and D4 in toxicity tests were determined by interpolation using their corresponding donor concentrations in toxicity tests and the best fit model determined for each chemical in the exposure confirmation experiment (Equation 1). The chemical activity measurements were due to technical reasons performed at $35^{\circ} \mathrm{C}$, and thus at a temperature somewhat higher than the toxicity test temperature of $20^{\circ} \mathrm{C}$. The higher temperature will lead to higher headspace concentrations but should not have a marked impact on the chemical activity calibration curves. Headspace measurements above donor solutions and pure substance references were done at the same 
temperature, and we assume the temperature effect on vaporization of the pure chemical in liquid form to be similar to the temperature effect on the lipid-gas phase transfer of that chemical.

The observed toxicity was linked to concentrations in the donor $\left(C_{\text {Donor, }}\right.$ Equation 2$)$ and also to calculated

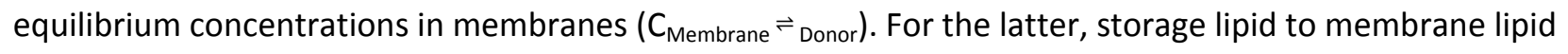
partition coefficients $K_{\mathrm{LM}}$ were first calculated as ratio of $K_{\mathrm{LW}}$ and $K_{\mathrm{MW}}$ (Table 1), and then used to determine

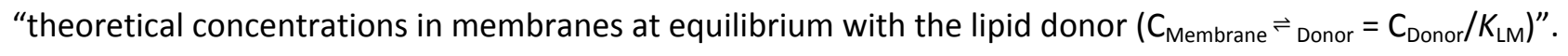
These conversions were not done for $\mathrm{C}_{\text {Donor }}>30 \%$, where headspace GC-MS results indicated non-linear partitioning. For test chemicals without clear toxicity at $C_{\text {Donor }}=30 \%$, no dose-response curves were thus produced.

\section{RESULTS AND DISCUSSION}

\subsection{Confirmation of test chemical exposures}

The measurements of headspace concentration and chemical activity were performed for limonene, nnonane, and octamethylcyclotetrasiloxane (D4), which represent the three groups of test chemicals: terpenes, alkanes, and siloxanes, respectively. The same trend was observed for all three chemicals. At low mass concentrations, the partitioning of test chemicals between Miglyol and air was proportional and thus followed as expected Henry's law where partition coefficients apply (Figure 1$)^{18}$. At higher $\mathrm{C}_{\text {Donor }}$ of typically 20-30\%, the partitioning became non-linear and entered the Raoult's law regime. At the highest donor concentrations, the chemical activity measurements clearly showed a deviation from ideal Raoult's law behavior, with chemical activity already reaching unity at mass concentrations below $100 \%$ (e.g., $80 \%$ )

(Figure 1). The best explanation for this deviation is that the tested chemicals were only fully miscible with Miglyol oil until a certain concentration, above which the concentrations in the headspace were controlled only by pure test substance. It was difficult to visually confirm a physical separation of the high concentration donor solutions into two phases, and the headspace concentration measurements were then instrumental for identifying this phenomenon. Additionally, these measurements provided a solid link 
between mass concentration and chemical activity, which in turn provided a defined exposure metric for the assessment of toxicity. Low standard error of the mean (SEM) values for replicate vials confirmed wellcontrolled exposure, and the high coefficients of determination $\left(r^{2}\right)$ led to well-defined exposure via interpolation (Figure 1).

Analytical confirmation of exposure in toxicity tests is essential for reliable toxicity assessments. For a test system using passive dosing with a dominant donor, it is usually sufficient to perform the exposure confirmation at the end of the test or in a separate test without the test organisms. Indeed, passive dosing with a dominant reservoir of test chemical in the donor can buffer losses during testing ${ }^{7,20,39-41}$. As shown in Table 1 , chemicals with high $K_{\text {AW }}$ values distribute predominantly (>90\%) to the headspace of a closed test system without a passive dosing donor. In this scenario, a majority of test chemical thus partitions into the headspace after being introduced into the aqueous test medium ${ }^{3,42}$, which in aquatic toxicity tests can lead to un-controlled exposure and severe underestimation of toxicity². In a passive dosing test system, the test chemicals are contained predominantly in the donor, e.g., >95\% (Table 1), which then via equilibrium partitioning will buffer any losses in the test medium and thus maintain the exposure. The mass balance calculations demonstrate the much higher test substance masses and concentrations in the headspace compared to the water, which make exposure confirmation via the headspace more straightforward and meaningful. We have in the present study also tried to confirm the exposure in the water, which turned out to be very difficult for VHOCs contained in these small volumes of water. Isooctane was the only test substance with a lipid to air partition coefficient $K_{\mathrm{LA}}<1000$, the only chemical with a donor depletion $>10 \%$, and thus omitted from all further data analysis.

To assess whether a test chemical exerts baseline or excess toxicity, external exposure concentrations can be converted to internal exposure metrics such as lipid normalized concentration, calculated equilibrium concentration in lipid, or chemical activity ${ }^{20,27,28}$. In this regard, the HS-PD from a lipid-based donor has a number of advantages. First, the observed toxicity can be linked directly to the test concentrations in the lipid donor for a simplified baseline toxicity assessment. Second, concentrations in the donor can be 
converted to calculated equilibrium concentrations in membranes $\left(C_{\text {Membrane }} \overrightarrow{\text { Donor }}_{\text {or }}\right)$, premised that required storage lipid-membrane lipid partition coefficient values are available, ${ }^{36}$ and effective concentrations can then be compared directly to the critical membrane concentration of $40-160 \mathrm{mmol} / \mathrm{kg}$. Third, the combination of HS-PD and HS-GCMS allows control and measurement of chemical activity in the test system. Measuring head space concentrations and chemical activity is essential for VHOCs, especially when it is challenging to measure their concentrations in the aqueous medium or in the test organism. The measurement is simple, and one can obtain precise data by utilizing fully automated analytical instruments. This again provides a solid exposure metric to be linked to the observed toxicity.

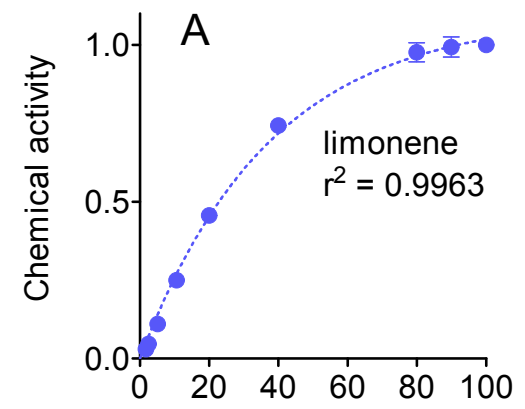

Mass concentration (\%)

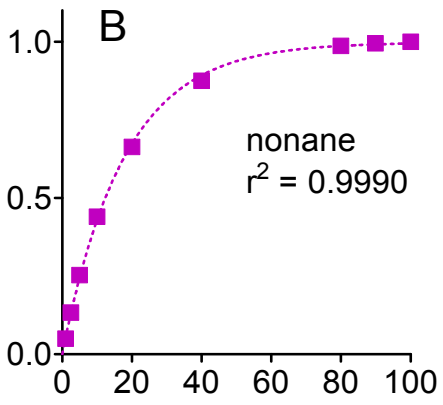

Mass concentration (\%)

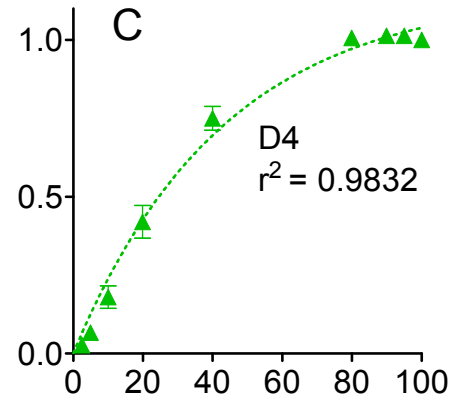

Mass concentration (\%)

Figure 1: Chemical activity of limonene (A), n-nonane (B), and octamethylcyclotetrasiloxane D4 (C) measured at different mass concentrations in Miglyol oil (mean \pm SEM, $n=3$ ).

\subsection{Toxicity test results}

The toxic effects of limonene, n-nonane, D4, and D5 on algae and springtail were examined both on a lipid basis and a chemical activity basis (Figure 2 and 3), whereas the toxic effects of other test chemicals were examined on the lipid basis only (Figure S3 and S4). Table S1 summarizes lipid based median effective concentrations (EC-50s) and Table S2 median effective chemical activities (Ea-50s) of limonene, n-nonane, and D4 in toxicity tests with both algae and springtails.

Figure 2A shows the growth inhibition of $R$. subcapitata after 72-h exposure to limonene, n-nonane, D4, and D5 against their concentrations in the lipid donor (Equation 2). The siloxanes exhibited no (by D5) or 
307 limited growth inhibition (11\% by D4) toward $R$. subcapitata as reported by Trac and colleagues ${ }^{8}$, and thus no EC-50 or Ea-50 values were derived for siloxanes in the algal growth inhibition tests. Figure $2 \mathrm{~B}$ shows the dose-response curves of limonene and n-nonane on the membrane basis, which is within and slightly above the grey box depicting the concentration range for baseline toxicity. Their EC-50 values were 210 and 130 $\mathrm{mmol} / \mathrm{kg}$ lipid, respectively (Table S1). This indicates that these two chemicals were baseline toxic towards algae and springtails and thus no excess toxicity was observed.

Figures $2 \mathrm{C}$ and $2 \mathrm{D}$ show the springtail lethality after 7-d exposure to limonene, nonane, D4, and D5 against their concentrations in the lipid donor and in the membranes at equilibrium with the donor. On the membrane basis, the EC-50 values were $90,90,70$, and $70 \mathrm{mmol} / \mathrm{kg}$, respectively (Table S1). For the other test chemicals, the EC-50 values in all toxicity tests with both algae and springtail were within or above the baseline toxicity range (Figure S4 and Table S1). These findings support the hypothesis that these test chemicals did not exert excess toxicity.

When assessed on the chemical activity basis, the Ea-50 values for limonene and nonane in the algal growth inhibition tests were 0.25 and 0.40 , respectively (Table S1), whereas the Ea-50 values for limonene, nonane, and D4 in the springtail lethality tests were $0.11,0.30$, and 0.42 , respectively (Table S1). Recent studies on algal growth inhibition caused by a large number of chemicals have reported that for nonpolar narcotic chemicals baseline toxicity requires $1 \%$ of saturation, i.e., chemical activity of $0.01^{21}$, whereas to initiate above the chemical activity range of 0.01-0.1 for baseline toxicity (Figure 3), thus supporting the absence of excess toxicity by these chemicals. 
R. subcapitata
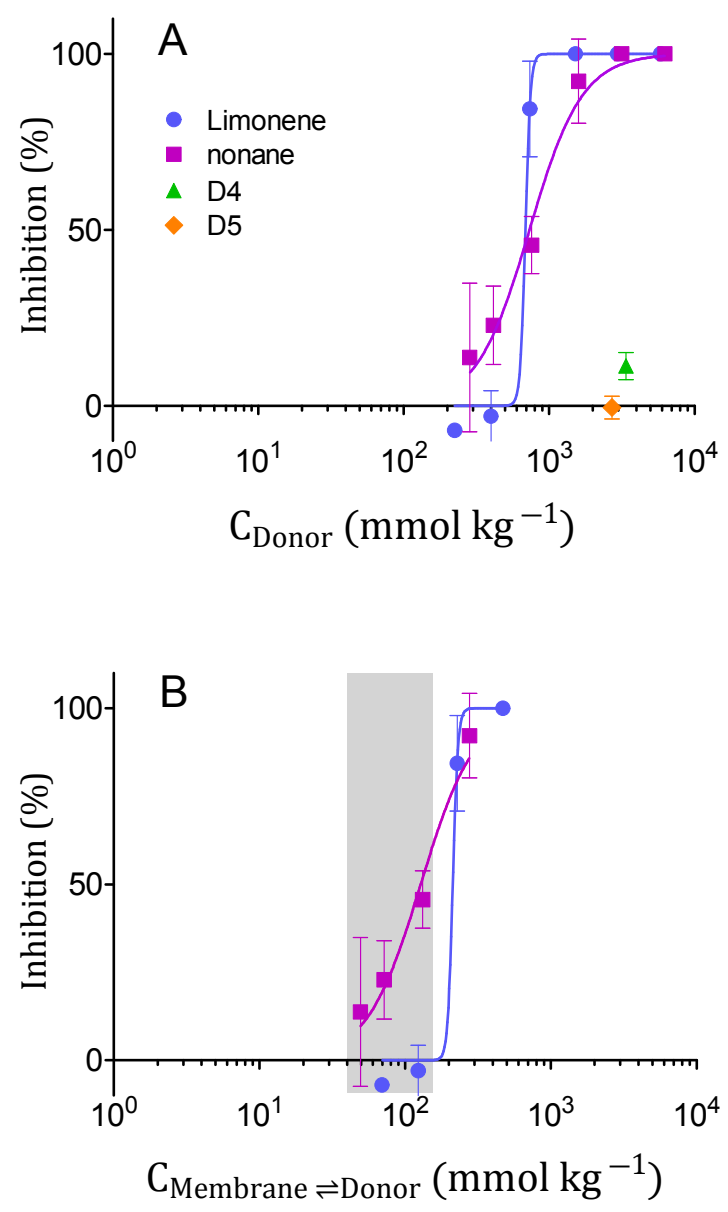

F. candida

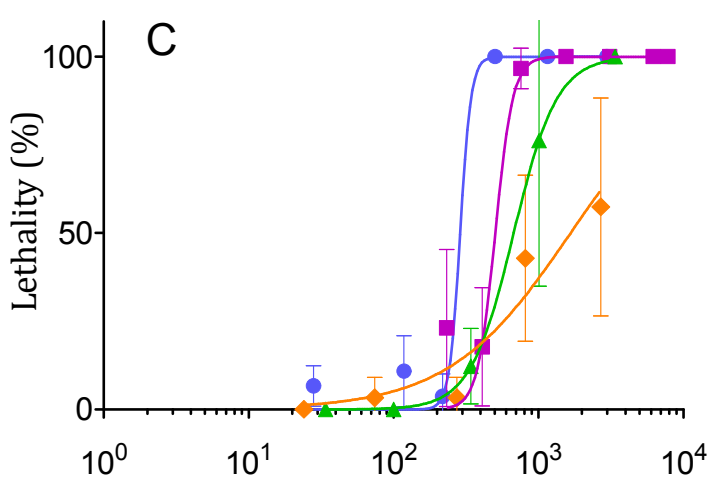

$\mathrm{C}_{\text {Donor }}\left(\mathrm{mmol} \mathrm{kg}^{-1}\right)$

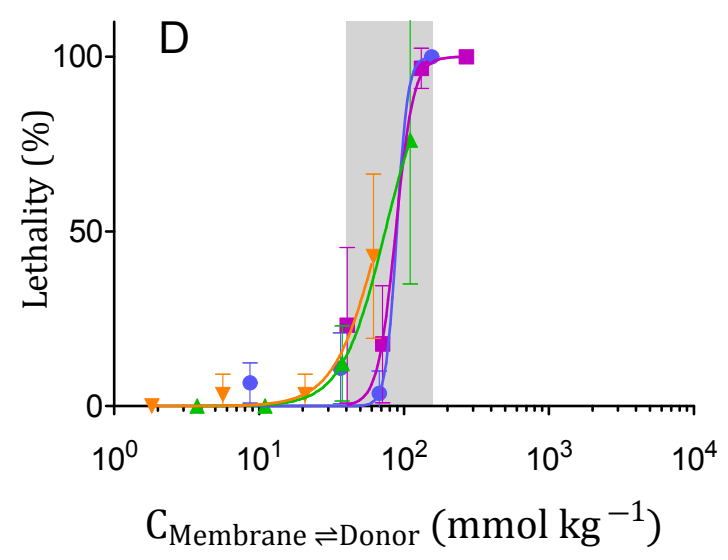

Figure 2: Toxicity of limonene, n-nonane, octamethylcyclotetrasiloxane (D4), and decamethylcyclopentasiloxane (D5) to algae and springtail linked to concentration in lipid donor (A \& C) and concentration in membranes at equilibrium with the donor ( $B$ \& $D)$ (mean $\pm S D, n=3$ ). Data for $D 4$ and D5 in algal growth inhibition test were from Trac et al. ${ }^{8}$. The grey boxes depict the reported concentration range of $40-160 \mathrm{mmol} / \mathrm{kg}$ membrane lipid for baseline toxicity.

For each test chemical toxicity was higher in springtail than algae (Table S1 and S2, Figure 2 and 3).

Remarkably, the springtail lethality at saturation was $100 \%$ for D4 and about 50\% for D5, which is markedly higher than the corresponding algal growth inhibition of D4 (11\% at saturation) and D5 (no inhibition) ${ }^{8}$ These toxicity differences for the siloxanes between the air-exposed springtail and the water-exposed algae can be explained by differences in the mass transfer of these chemicals through air and water. Indeed, the 
341 bioconcentration kinetics of hydrophobic organic chemicals in aquatic organisms is generally rate limited by 342 molecular diffusion through an unstirred aqueous boundary layer (UBL) ${ }^{43}$. Mayer and colleagues ${ }^{4}$ 343 demonstrated that the diffusive mass transfer of 2-3 ringed PAHs was higher through air than through water. This observation is consistent with the observation by Hanzel and colleagues ${ }^{5,6}$, who demonstrated that diffusive mass transfer of naphthalene is more efficient through soil interstitial air compared to soil interstitial water, and that air-exposure consequently can drive the naphthalene bioavailability to soil bacteria. Both observations can be explained by a higher product of diffusion coefficient and UBL concentration gradient for 2-3 ringed PAHs in air compared to water. The test chemicals in the present study have much higher air to water partition coefficients $\left(K_{\mathrm{AW}}\right)$ compared to PAHs. Hence, we expected much higher diffusive mass transfer and thus faster uptake kinetics for air compared to water exposure, which is consistent with the observed presence of toxicity in springtails and absence of toxicity in algae. $\left.3.13^{44}\right)$. Their high hydrophobicity leads also to high concentration and retention in waste water treatment plant (WWTP) sludge, soils, and sediments, and the high $K_{\mathrm{AW}}$ values make air-exposure crucial for soil dwelling organisms, particularly in WWTP sludge amended soil. The combination of headspace passive dosing and the springtail lethality test can then be a simple and efficient way to link such soil air-exposure to toxicological endpoints.

R. subcapitata

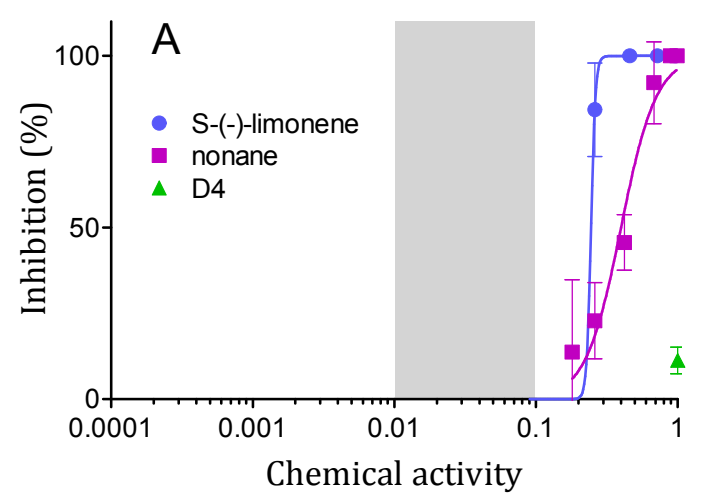

F. candida

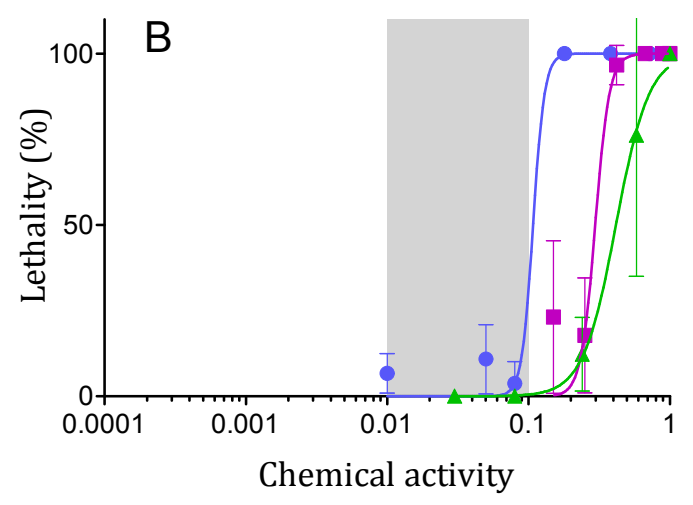


Figure 3: Toxicity of limonene, nonane, and octamethyltetracyclosiloxane (D4) to algae (A) and springtail (B) linked to chemical activity (mean $\pm S D, n=3$ ). Data for $D 4$ in algal growth inhibition test were from Trac et al. (mean $\pm S D, n=6)^{8}$. The grey boxes depict the reported chemical activity range of 0.01-0.1 for baseline toxicity.

The HS-PD method developed in the present study is an effective method that enables the dose-response testing of liquid VHOCs and provides also an efficient way to assess their inherent toxicity by linking toxicity to estimated equilibrium concentrations in membranes. We expect the method to be applicable to chemicals with a sufficiently high air-water partition coefficient to allow equilibration via the headspace. All test chemicals in the present study have $K_{\mathrm{AW}}>1 \mathrm{~L} / \mathrm{L}$, while other headspace passive dosing studies included semivolatile organic chemicals with lower $K_{\mathrm{Aw}}{ }^{31-33}$. Further, the test chemicals need as a basic rule $K_{\mathrm{LW}}>$ $1000 \mathrm{~L} / \mathrm{L}$ and $K_{\mathrm{LA}}>1000 \mathrm{~L} / \mathrm{L}$ in order to avoid donor depletion. Headspace passive dosing seems also suited for chronic and even multi-generation ecotoxicity testing with small aquatic and terrestrial animals ${ }^{41,45}$, since it circumvents biofilm formation on the passive dosing donor (i.e., no direct contact with medium) during extended test durations. Such chronic and multi-generation studies are particularly needed for highly hydrophobic chemicals that are prone to slow bioconcentration kinetics, even for the small test organisms used in the present study.

The loading of the passive dosing donor is one of the most time consuming and critical steps in passive dosing. A procedural novelty in the present work is the preparation of the donor by simply adding the liquid test chemical to a liquid donor lipid. This allows setting up the test systems for a reasonably large number of chemicals, treatments and replicates to be tested without significant delay. A main limitation of using a lipid donor has been that we could not find a lipid that is practical (i.e., high capacity, low viscosity liquid, clean, and good commercial availability) and at the same time is the optimal surrogate for the phospholipid membrane. The Miglyol oil used in the present study was practical and provided excellent passive dosing performance, and the subsequent conversions using estimated $K_{\mathrm{LM}}$ values were also found a practical and effective way to correct for partitioning differences between storage and membrane lipids. 
384 The new combination of exposure control and analytical exposure confirmation for VHOCs can provide 385 sound toxicity data, which now can be further assessed, applied to models, and used in risk assessment. 386 Specifically, the headspace concentration measurements above the lipid donor provided an analytical 387 calibration between equilibrium concentrations in lipids and the thermodynamic chemical activity (Figure 388 1). In the present study, both exposure metrics were used to assess VHOC toxicity and led to the same 389 results and conclusions.

\section{ASSOCIATED CONTENT}

\section{Supporting information}

392 393 394 395

Additional figures for the chemical activity measurements, algal growth in the controls in algal inhibition tests, toxicity of other test chemicals assessed on the lipid basis, and additional tables for toxicity values and dose-response data.

\section{ACKNOWLEDGEMENTS}

We acknowledge the analytical support from Hanne Bøgild (DTU) and the maintenance of springtail culture from Elin Jørgensen and Zdenek Gavor (AU). Lam N. Trac acknowledges the PhD funding from DTUEnvironment, and we thank for additional funding from the CEFIC LRI project ECO 42: Fate-directed toxicity testing and risk assessment of UVCBs. We thank the reviewers for their constructive criticism and input.

\section{REFERENCES}

(1) OECD. Guidance Document on Aquatic Toxicity Testing of Difficult Substances and Mixtures. Series on Testing and Assessment No. 23 (2nd edition). Organ. Econ. Co-operation Dev. 2019, 23, 1-81.

(2) Mayer, P.; Nyholm, N.; Verbruggen, E. M. J.; Hermens, J. L. M.; Tolls, J. Algal growth inhibition test in filled, closed bottles for volatile and sorptive materials. Environ. Toxicol. Chem. 2000, 19 (10), 25512556. 


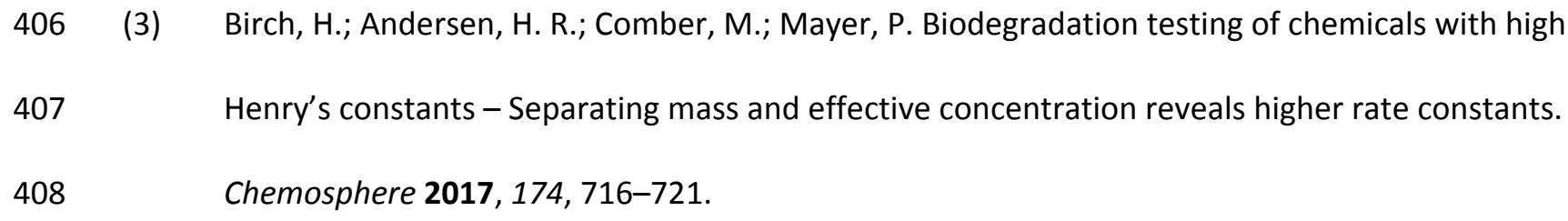

(4) Mayer, P.; Fernqvist, M. M.; Christensen, P. S.; Karlson, U.; Trapp, S. Enhanced diffusion of polycyclic aromatic hydrocarbons in artificial and natural aqueous solutions. Environ. Sci. Technol. 2007, 41 (17), 6148-6155.

(5) Hanzel, J.; Harms, H.; Wick, L. Y. Bacterial Chemotaxis along Vapor-Phase Gradients of Naphthalene. Environ. Sci. Technol. 2010, 44 (24), 9304-9310.

(7) Smith, K. E. C.; Dom, N.; Blust, R.; Mayer, P. Controlling and maintaining exposure of hydrophobic organic compounds in aquatic toxicity tests by passive dosing. Aquat. Toxicol. 2010, 98 (1), 15-24.

(8) Trac, L. N.; Schmidt, S. N.; Mayer, P. Headspace passive dosing of volatile hydrophobic chemicals Aquatic toxicity testing exactly at the saturation level. Chemosphere 2018, 211, 694-700.

(9) McCarty, L. S.; Mackay, D. Enhancing ecotoxicological modeling and assessment - Body residues and modes of toxic action. Environ. Sci. Technol. 1993, 27 (9), 1718-1728.

(10) van Wezel, A. P.; Opperhuizen, A. Narcosis due to environmental pollutants in aquatic organisms: residue-based toxicity, mechanisms, and membrane burdens. Crit. Rev. Toxicol. 1995, 25 (3), 255279.

(11) Mayer, P.; Halling-Sørensen, B.; Sijm, D. T. H. M.; Nyholm, N. Toxic cell concentrations of three polychlorinated biphenyl congeners in the green alga Selenastrum capricornutum. Environ. Toxicol. Chem. 1998, 17 (9), 1848-1851. 
428 (12) Di Toro, D. M.; McGrath, J. a; Hansen, D. J. Technical basis for narcotic chemicals and polycyclic aromatic hydrocarbon criteria. I. Water and tissue. Environ. Toxicol. Chem. 2000, 19 (8), 1951.

(13) Van Der Heijden, S. A.; Hermens, J. L. M.; Sinnige, T. L.; Mayer, P.; Gilbert, D.; Jonker, M. T. O. Determining high-quality critical body residues for multiple species and chemicals by applying improved experimental design and data interpretation concepts. Environ. Sci. Technol. 2015, 49 (3), 1879-1887.

(14) Ferguson, J. The Use of Chemical Potentials as Indices of Toxicity. Proc. R. Soc. B Biol. Sci. 1939, 127 (848), 387-404.

(15) Mayer, P.; Reichenberg, F. Can highly hydrophobic organic substances cause aquatic baseline toxicity and can they contribute to mixture toxicity? Environ. Toxicol. Chem. 2006, 25 (10), 26392644.

(16) Reichenberg, F.; Mayer, P. Two complementary sides of bioavailability: accessibility and chemical activity of organic contaminants in sediments and soils. Environ. Toxicol. Chem. 2006, 25 (5), 12391245.

(17) Gobas, F. A. P. C.; Mayer, P.; Parkerton, T. F.; Burgess, R. M.; van de Meent, D.; Gouin, T. A chemical activity approach to exposure and risk assessment of chemicals. Environ. Toxicol. Chem. 2018, 37 (5),

(18) Schwarzenbach, R. P.; Gschwend, P. M.; Imboden, D. M. Environmental Organic Chemistry, 2nd ed.; John Wiley \& Sons, Inc.: Hoboken, NJ, USA, 2002.

(19) Mackay, D.; Arnot, J. A.; Petkova, E. P.; Wallace, K. B.; Call, D. J.; Brooke, L. T.; Veith, G. D. The physicochemical basis of QSARs for baseline toxicity. SAR QSAR Environ. Res. 2009, 20 (3-4), 393414. 


\begin{abstract}
450 (20) Schmidt, S. N.; Smith, K. E. C.; Holmstrup, M.; Mayer, P. Uptake and toxicity of polycyclic aromatic hydrocarbons in terrestrial springtails-studying bioconcentration kinetics and linking toxicity to chemical activity. Environ. Toxicol. Chem. 2013, 32 (2), 361-369.
\end{abstract}

(21) Schmidt, S. N.; Mayer, P. Linking algal growth inhibition to chemical activity: Baseline toxicity required $1 \%$ of saturation. Chemosphere 2015, 120, 305-308.

(22) Mackay, D.; Arnot, J. A.; Wania, F.; Bailey, R. E. Chemical activity as an integrating concept in environmental assessment and management of contaminants. Integr. Environ. Assess. Manag. 2011, $7(2), 248-255$.

(23) Schmidt, S. N.; Armitage, J. M.; Arnot, J. A.; Mackay, D.; Mayer, P. Linking algal growth inhibition to chemical activity: Excess toxicity below $0.1 \%$ of saturation. Chemosphere 2018, 208, 880-886.

(24) Mayer, P.; Holmstrup, M. Passive dosing of soil invertebrates with polycyclic aromatic hydrocarbons: Limited chemical activity explains toxicity cutoff. Environ. Sci. Technol. 2008, 42 (19), 7516-7521.

(25) Schmidt, S. N.; Holmstrup, M.; Smith, K. E. C.; Mayer, P. Passive dosing of polycyclic aromatic hydrocarbon $(\mathrm{PAH})$ mixtures to terrestrial springtails: Linking mixture toxicity to chemical activities, equilibrium lipid concentrations, and toxic units. Environ. Sci. Technol. 2013, 47 (13), 7020-7027.

(26) Smith, K. E. C.; Oostingh, G. J.; Mayer, P. Passive dosing for producing defined and constant exposure of hydrophobic organic compounds during in vitro toxicity tests. Chem. Res. Toxicol. 2010,

(27) Smith, K. E. C.; Schmidt, S. N.; Dom, N.; Blust, R.; Holmstrup, M.; Mayer, P. Baseline toxic mixtures of non-toxic chemicals: "solubility addition" increases exposure for solid hydrophobic chemicals. 

high throughput passive dosing format for the Fish Embryo Acute Toxicity test. Chemosphere 2015, 139, 9-17.

(29) Butler, J. D.; Parkerton, T. F.; Redman, A. D.; Letinski, D. J.; Cooper, K. R. Assessing AromaticHydrocarbon Toxicity to Fish Early Life Stages Using Passive-Dosing Methods and Target-Lipid and Chemical-Activity Models. Environ. Sci. Technol. 2016, 50 (15), 8305-8315.

(30) Bragin, G. E.; Parkerton, T. F.; Redman, A. D.; Letinksi, D. J.; Butler, J. D.; Paumen, M. L.; Sutherland, C. A.; Knarr, T. M.; Comber, M.; den Haan, K. Chronic toxicity of selected polycyclic aromatic hydrocarbons to algae and crustaceans using passive dosing. Environ. Toxicol. Chem. 2016, 35 (12), $2948-2957$.

(31) Jahnke, A.; Mayer, P. Do complex matrices modify the sorptive properties of polydimethylsiloxane (PDMS) for non-polar organic chemicals? J. Chromatogr. A 2010, 1217 (29), 4765-4770.

(32) Jahnke, A.; Holmbäck, J.; Andersson, R. A.; Kierkegaard, A.; Mayer, P.; MacLeod, M. Differences between Lipids Extracted from Five Species Are Not Sufficient To Explain Biomagnification of Nonpolar Organic Chemicals. Environ. Sci. Technol. Lett. 2015, 2 (7), 193-197.

(33) Bolinius, D. J.; MacLeod, M.; ladaresta, F.; Holmbäck, J.; Jahnke, A. Sorptive Capacities of Nonpolymeric Plant Lipids for Hydrophobic Chemicals Determined by Passive Dosing. Environ. Sci. Technol. 2019, 53 (3), 1278-1286.

(34) Wang, Y.; O’Reilly, J.; Chen, Y.; Pawliszyn, J. Equilibrium in-fibre standardisation technique for solidphase microextraction. J. Chromatogr. A 2005, 1072 (1), 13-17.

(35) Zhao, W.; Ouyang, G.; Pawliszyn, J. Preparation and application of in-fibre internal standardization solid-phase microextraction. Analyst 2007, 132 (3), 256.

(36) Helmholz Centre for Environmental Research - UFZ. UFZ - LSER Database 


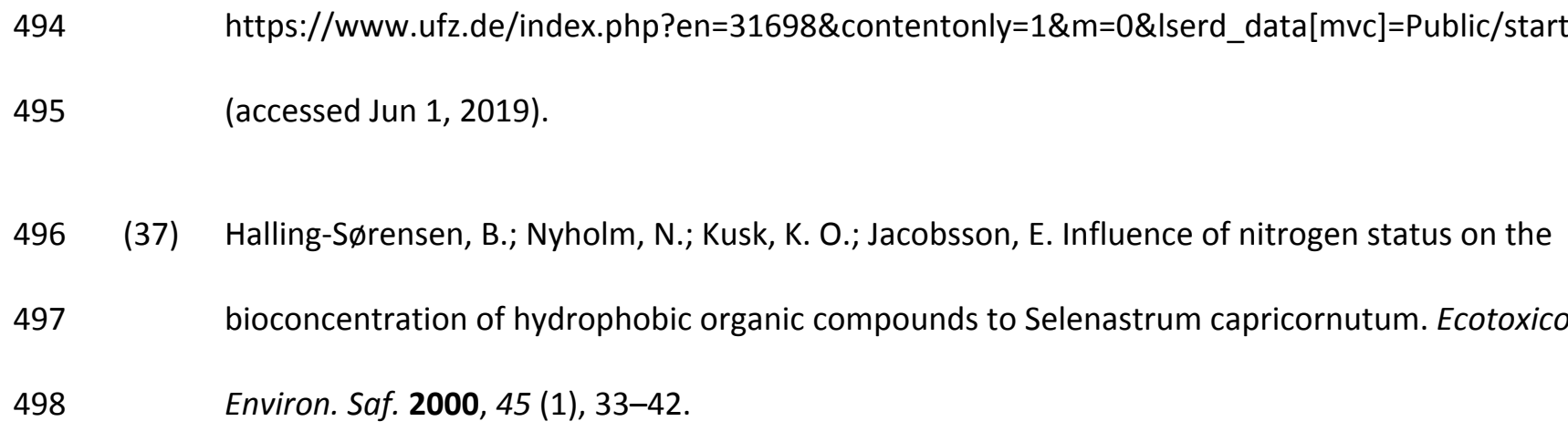

(37) Halling-Sørensen, B.; Nyholm, N.; Kusk, K. O.; Jacobsson, E. Influence of nitrogen status on the

(39) Butler, J. D.; Parkerton, T. F.; Letinski, D. J.; Bragin, G. E.; Lampi, M. A.; Cooper, K. R. A novel passive dosing system for determining the toxicity of phenanthrene to early life stages of zebrafish. Sci. Total Environ. 2013, 463-464, 952-958.

(40) Smith, K. E. C.; Jeong, Y.; Kim, J. Passive dosing versus solvent spiking for controlling and maintaining hydrophobic organic compound exposure in the Microtox?? assay. Chemosphere 2015, 139, 174180.

(41) Ribbenstedt, A.; Mustajärvi, L.; Breitholtz, M.; Gorokhova, E.; Mayer, P.; Sobek, A. Passive dosing of triclosan in multigeneration tests with copepods - stable exposure concentrations and effects at the low $\mu$ g/L range. Environ. Toxicol. Chem. 2017, 36 (5), 1254-1260.

(42) Birch, H.; Hammershøj, R.; Mayer, P. Determining biodegradation kinetics of hydrocarbons at low concentrations: Covering 5 and 9 orders of magnitude of Kow and Kaw. Environ. Sci. Technol. 2018, $52(4), 2143-2151$.

(43) Bayen, S.; ter Laak, T. L.; Buffle, J.; Hermens, J. L. M. Dynamic Exposure of Organisms and Passive Samplers to Hydrophobic Chemicals. Environ. Sci. Technol. 2009, 43 (7), 2206-2215. 
Superhydrophobic Chemicals: A Modeling Case Study of Cyclic Volatile Methyl Siloxanes. Environ. Sci. Technol. 2015, 49 (19), 11913-11922.

(45) Leon Paumen, M.; Steenbergen, E.; Kraak, M. H. S.; van Straalen, N. M.; van Gestel, C. A. M. Multigeneration Exposure of the Springtail Folsomia candida to Phenanthrene: From 6985-6990. 